\title{
The regulation of positive and negative social feedback: A psychophysiological study
}

\author{
Marie-Anne Vanderhasselt • Jonathan Remue • \\ Kwun Kei Ng • Sven C. Mueller • Rudi De Raedt
}

Published online: 26 March 2015

(C) Psychonomic Society, Inc. 2015

\begin{abstract}
Everyday social evaluations are psychologically potent and trigger self-reflective thoughts and feelings. The present study sought to examine the psychophysiological impact of such evaluations using eye tracking, pupillometry, and heart-rate variability. Fifty-nine healthy adult volunteers received rigged social feedback (criticism and praise) based on their photograph. Gaze data were collected to investigate processes of attentional deployment/allocation toward the self or the evaluator expressing criticism or praise. Whereas voluntary attention was directed to evaluators who expressed praise, attention was drawn to one's own picture after criticism. Pupil dilation and heart-rate variability were larger in response to criticism as compared to praise, suggesting a flexible and adaptive emotion regulatory effort in response to social information that triggers an affective response. Altogether, healthy individuals recruited more regulatory resources to cope with negative (as compared to positive) social feedback, and this processing of social feedback was associated with adjustments in self-focused attention.
\end{abstract}

Keywords Social feedback · Pupil dilation .

Heart-rate variability $\cdot$ Gaze patterns

Electronic supplementary material The online version of this article (doi:10.3758/s13415-015-0345-8) contains supplementary material, which is available to authorized users.

M.-A. Vanderhasselt $(\varangle) \cdot J$. Remue $\cdot$ S. C. Mueller $\cdot$ R. De Raedt Department of Experimental Clinical and Health Psychology, Ghent University, Henri Dunantlaan 2, 9000 Ghent, Belgium e-mail: MarieAnne.Vanderhasselt@Ugent.be

\section{K. K. Ng}

Department of Psychology, National University of Singapore,

9 Arts Link, Singapore 117570, Singapore
In society, it is a fundamental human need to belong to, and to be accepted by, the people around us. This basic need drives social bonding and the forming of attachments, interactions, and relationships (Baumeister \& Leary, 1995). As a consequence, being rejected, excluded or criticized is a distressing experience and activates self-conscious emotions (e.g., feeling hurt) and self-related thoughts. Fortunately, healthy individuals can regulate these rejection-induced thoughts and feelings to protect their self-esteem and maintain emotional well-being. On a theoretical level, Gross and Thompson (2007) postulated that individuals use attentional deployment to direct attention towards or away from the emotion-eliciting aspects of the situation. This way, attention allocation is one of the first information-processing steps of how individuals handle or regulate the generation of emotional responses in reaction to distressing information and challenging contexts (Gross \& Thompson, 2007).

Measuring eye movements to track visual gaze is a key method for assessing visual attention and attention allocation to affective stimuli. Interestingly, a relatively small number of studies investigated attentional deployment in response to social feedback. Within the social (rejection) context, prior eyetracking studies have mainly investigated averted/directed eye gaze as a nonverbal form of ostracism (e.g., Wirth, Sacco, Hugenberg, \& Williams, 2010) or to emotional faces after (threat of) social exclusion (e.g., DeWall, Maner, \& Rouby, 2009). Silk and colleagues (2012) investigated visual gazes during a naturalistic viewing condition after being included or excluded from a chat game, specifically focusing on the attentional allocation towards self-relevant information. However, these authors only examined visual gazes towards the participant's own photograph and another person receiving opposite feedback, whereas selective attention towards the photograph of the evaluator who provided the social feedback was not analyzed. Yet, the allocation of attention towards the evaluator versus the self following emotional stressors may inform on the impact of social feedback on information 
processes supporting emotion regulation. Because (1) social rejection is known to induce levels of negative affect (e.g., Blackhart, Nelson, Knowles, \& Baumeister, 2009; Leary, Koch, \& Hechenbleikner, 2001) and (2) negative affect is associated with increased self-focused thoughts and attention to negative self aspects (Mor \& Winquist, 2002), negative social feedback might increase attention allocation to one's own portrait photograph. On the other hand, as positive social feedback induces positive affect (Blackhart et al., 2009), this type of feedback could facilitate approach behaviors that motivate people to engage, explore and interact with their environment in a variety of ways (Fredrickson, 1998). Therefore, positive social feedback could be associated with attention allocation towards the evaluator providing feedback.

In line with the conceptualization of Gross and Thompson (2007), distinguishing between attentional deployment and cognitive change processes in emotion regulation, we used (a) gaze tracking to measure the attentional deployment component of emotion regulation, and (b) two physiological indices of effortful regulatory responses following socially stressful stimuli. In this study, pupil dilation and heart-rate variability (HRV) are assessed. Pupil dilation has been associated with emotional information processing and arousal (e.g., Bradley, Miccoli, Escrig, \& Lang, 2008), likely reflecting limbic reactivity (e.g., Siegle, Steinhauer, Stenger, Konecky, \& Carter, 2003; Siegle, Steinhauer, Thase, Stenger, \& Carter, 2002). Moreover, based on the link between arousal and mental effort (Van Steenbergen \& Band, 2013), pupillary activity has been proposed as a physiological marker of cognitive effort required to control (automatic) emotional responses, likely reflecting activity in prefrontal regions (e.g., Siegle et al., 2003; Siegle, Steinhauer, Friedman, Thompson, \& Thase, 2011; Urry et al., 2006; van Reekum et al., 2007). Therefore, pupil dilation can be considered an indicator of dynamic changes in cognitive-affective brain activity (Critchley, Tang, Glaser, Butterworth, \& Dolan, 2005; Siegle et al., 2003; Urry, van Reekum, Johnstone, \& Davidson, 2009), likely reflecting the level of central nervous processing allocated to regulate emotional responses.

Heart-rate variability (HRV), another peripheral physiological index of emotion regulation, is an indirect measure of parasympathetic (vagal) control over fluctuations in the length of the interbeat interval (e.g., Park, Vasey, Van Bavel, \& Thayer, 2014). HRV is considered a physiological index of flexible adaptation to environmental demands and emotional situations, such as worry to emotional film clips (e.g., Aldao, Mennin, \& McLaughlin, 2013). A number of studies have identified a positive association between adaptive regulatory functioning (in contrast to rigid, hyper-vigilant responses) and (1) resting (tonic) HRV (e.g., Koval et al., 2013; Woody, McGeary, \& Gibb, 2014; for a review, see Appelhans \& Luecken, 2006) and (2) phasic HRV cardiac vagal activity (the change from rest to task; for a review, see Thayer, Åhs,
Fredrikson, Sollers, \& Wager, 2012). Even though some inconsistencies exist (e.g., Rottenberg, 2007), meta-analyses and systematic reviews propose both higher tonic and phasic HRV to be a physiological marker of emotion regulation and stress-adaptability (e.g., Park et al., 2014; Thayer et al., 2012; Thayer, Hansen, Saus-Rose, \& Johnsen, 2009; Thayer \& Lane, 2000). Moreover, depressive brooding, a maladaptive ruminative thinking style that plays a key role in the onset and maintenance of depression has been associated with lower tonic HRV (Woody et al., 2014). Similarly, HRV decreases in response to stress and worry (Delaney \& Brodie, 2009; Pieper, Brosschot, van der Leeden, \& Thayer, 2007).

Thus, the present study examined complementary psychophysiological markers that each inform about component processes of emotion regulation, including visual gaze (as a measure of attentional deployment), pupil dilation (as a measure of central nervous system processing of cognitive-affective brain processing), and HRV (as a measure of flexible adaptation to environmental stressors) to explicit social feedback. With regard to eye movements, we expect attention to be especially deployed towards the self after receiving negative social feedback (i.e., critique), but towards the evaluator after receiving positive social feedback (i.e., praise; Fredrickson, 1998; Mor \& Winquist, 2002). With regard to pupil dilation, we hypothesize greater dilation in response to negative as compared to positive feedback (Silk et al., 2012). With regard to the cardiac response, we predict increased HRV in response to social feedback, with a specific increase in blocks receiving predominantly negative (as compared to positive) social feedback (taken into account tonic HRV, e.g., Thayer et al., 2009; Thayer \& Lane, 2000). We expect depressive brooding, a maladaptive form of emotion regulation, to be inversely correlated to HRV - controlled for the tonic HRV at baselineduring the social feedback (Woody et al., 2014; Pieper et al., 2007). Because rumination has been found to be positively associated with gaze fixation and pupil dilation in the processing of emotional material (e.g., Duque, Sanchez, \& Vazquez, 2014), we will also explore the association between depressive brooding and both of these latter physiological indices.

\section{Method}

\section{Participants}

Fifty-nine healthy undergraduates of Ghent University (10 male, 49 female, age range $17-36$ years; $M=19.65, S D=3.65$ ) volunteered to participate for course credit. All participants were right-handed, with normal or corrected-to-normal vision, and none reported current or past history of neurological or psychiatric illness. Moreover, exclusion criteria consisted of reports of serious head injury or eye problems and visual difficulties not corrected by the use of glasses or contact lenses. Participants 
were recruited in the context of a larger project investigating other neurocognitive markers of emotion regulation and selfesteem, ${ }^{1}$ but participants were told this was a study on the development of first impressions. All participants provided written informed consent (protocol approved by the local ethics committee of Ghent University).

\section{Protocol}

Participants were told that this was a study on the development of first impressions. The paradigm consisted of three phases. The first two phases could be performed at home (communication via e-mail). In the first phase, approximately 2 weeks prior to the experiment, participants were asked to send a portrait photograph of themselves to the researcher. Participants were led to believe that their photograph would be sent to another participating university, where a panel of undergraduates would be forming first impressions of them during this interim period. In the second phase, after receiving their portrait picture, we sent them a series of 20 photographs of assumed participants from that other participating university, and our participants were asked, based on their first impression, to (1) indicate whether they liked or disliked that person, and (2) indicate in one or two words why they liked or disliked that person (open question). Only if participants completed the first two phases were they included in the third and last phase of the task that was conducted in the lab. In the last phase, all participants were tested individually. At the start of the experiment, participants were connected to the polar equipment (watch plus heart-rate monitor belt), and heart rate was collected during the whole experiment. Participants filled in the questionnaires on mood and trait rumination, and were subsequently asked to relax for 20 minutes. Afterwards, we repeated the cover story about the investigation of first impressions and then told the participants we were going to inform them about the first impressions provided by each of the other participating undergraduates: (1) whether they were liked or disliked by the others, and (2) why this was the case as described by one or two adjectives. We also told participants to concentrate on the evaluations because this might help them to answer questions afterwards to increase our understanding on how people use first impressions to decide whether or not they like someone. In reality, no social feedback was provided by other participants, but was rigged and carefully chosen in a controlled fashion (see description social feedback paradigm). After the social feedback paradigm, participants were asked to report their current mood state. Finally, participants were asked to rest for 15 minutes and were subsequently asked to

\footnotetext{
${ }^{1}$ The internal Shift Task, Implicit Association Task, and some other questionnaires (such as self esteem) were measured as well, but will not be reported in this manuscript.
}

report their level of state rumination during the latter rest period.

After the experiment, participants were asked whether they thought the first impressions were natural, and whether they believed the set-up. Thereafter, participants were debriefed about the real goal of this experiment, and the deceptive nature of the task was discussed with them.

\section{Social feedback paradigm}

We developed a paradigm to provide explicit feedback of other people about how desirable or likable individuals are based on their photograph. Facial stimuli were obtained by taking photographs of volunteers between the ages of 18 and 30 years, after these volunteers had given their written informed consent. Faces were age matched to the participants' age $(p>.5)$. Half of the faces were male, half of the faces were female. Everybody was asked to smile when taking the photograph. Both genders provided the same number of positive and negative feedback, and were presented equally to the left or right side of the screen. The feedback words were selected from a validated database of Hermans and De Houwer (1994), and matched on familiarity and word length. Moreover, positive $(M=4.86, S D=2.66)$ and negative $(M=4.31, S D=2.25)$ words, for positive and negative social feedback, respectively, were matched on arousal $(p=.37)$ based on the results of a small pilot study on 20 random people on our campus.

During the task, an experimental trial started with the presentation of a fixation point $(2,000 \mathrm{~ms})$ in the middle of the screen, and participants were asked to focus on this point, or return their gaze to this point when the faces disappeared from the screen. Subsequently, the face of the participant was presented together with the face of the evaluator $(5.9 \mathrm{~cm} \times 7.4 \mathrm{~cm}$ each face). The location of these two smiling faces (left or right) was counterbalanced, and each trial contained a portrait photograph of a different unfamiliar evaluator. Luminance and the size of both photographs was corrected to standardize these over the different conditions. After 3,000 ms, the socalled first impression appeared for 6,000 ms. This first impression consisted of an " $\mathrm{X}$ " (negative social feedback) or a "+" (positive social feedback) over the photograph of the participant, together with one or two words why the evaluator liked or disliked the participant (depicted under the photograph of the participant, Fig. 1).

The experimental task consisted of four blocks, each containing 18 trials (participants completed 72 trials in total), with a short break between the blocks. In two blocks, participants received mainly positive social feedback (12 out of 18 trials), whereas mainly negative social feedback ( 12 out of 18 trials) was presented in the two remaining blocks. Blocks were semicounterbalanced: Half of the participants started with a positive social feedback block and half started with a negative 


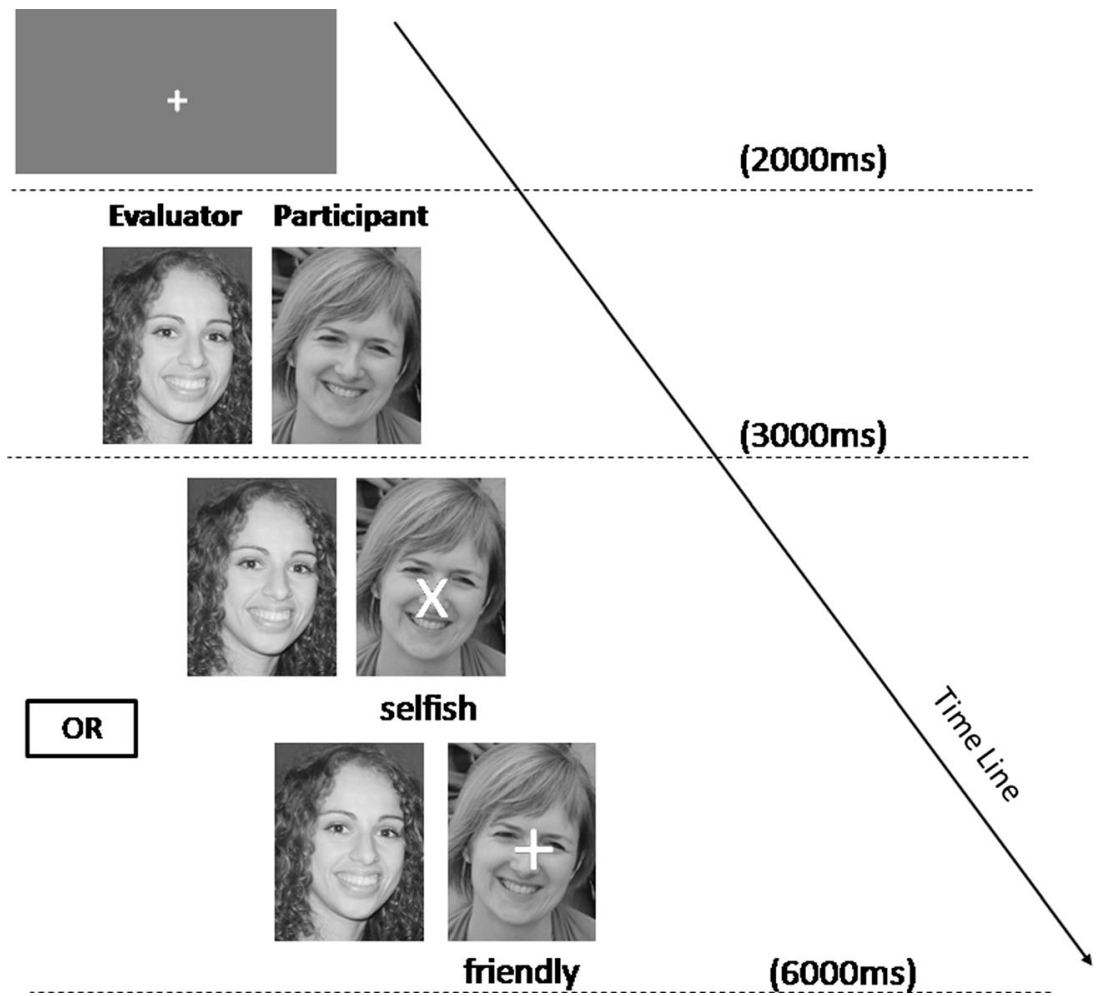

Fig. 1 Schematic overview of the social feedback paradigm. First, a fixation cross was presented in the center of the screen $(2,000 \mathrm{~ms})$, followed by the presentation of the portrait photograph of the participant together with the photograph of the evaluator (each trial a new evaluator, counterbalanced left/right location). After $3000 \mathrm{~ms}$, the

social feedback block, and the three subsequent blocks were alternating (positive vs. negative social feedback blocks).

Questionnaires

\section{Rumination}

1) Trait rumination was assessed using the Ruminative Responses Scale (RRS; Treynor, Gonzales, \& NolenHoeksema, 2003), which consists of 22 items that describe responses to a depressed mood that are focused on the self, symptoms, or consequences of the mood. A factor analysis of the RRS has identified a depressive brooding subscale (5 items) (Treynor et al., 2003). This subscale relates to a passive focus on one's problems, negative mood, and their consequences. The RRS can also be used to assess a measure of reflective pondering, which is, compared to depressive brooding, a more adaptive form of rumination. Higher scores indicate more rumination tendencies. Because the subscales scores of the RRS were not normally distributed (using the ShapiroWilk test), they were $\log$-transformed $(\log 10)$.

2) To obtain a state measure of ruminative thoughts following the social feedback task, we used a questionnaire that measures momentary self-reflective rumination (Mor, so-called first impression appeared for 6,000 ms. This first impression consisted of an "X" (dislike) or of a "+" (like) over the photograph of the participant, together with one or two words why the evaluator liked or disliked the participant (depicted under the photograph of the participant) (e.g., friendly, selfish)

Marchetti, \& Koster, 2015). All six questions relate to self-referent, ruminative thoughts as a particular selffocus on feelings, reactions, and sensations without immediate environmental demands. The statements are not inherently negative or positive, and are considered as a state measure of ruminative thinking (e.g. "Right now, I am thinking about how happy or sad I feel" and "Right now, I wonder why I react the way I do"; cf. Table supplementary material). Participants were requested to indicate whether they were engaging in these thoughts during the 10 minutes of rest. They were asked to respond using a seven-point Likert scale ranging from 1 (totally disagree) to 7 (totally agree) in order to measure the intensity of self-referent thinking.

Mood measures In order to evaluate temporary changes in mood before $\left(T_{\text {pre }}\right)$, versus immediately after $\left(T_{\text {post }}\right)$ the social feedback paradigm, mood ratings were administered using six visual analogue scales (VAS) providing measures of fatigue, tension, anger, vigor, depression, and cheerful mood (McCormack et al. 1988). Participants were asked to describe how they felt "at that moment" by indicating on horizontal $100 \mathrm{~cm}$ lines whether they experienced the five abovementioned mood states, from totally not to very much. 


\section{Eye tracker and HRV data acquisition}

Participants were tested in a quiet laboratory with dimmed light. For the acquisition of pupillary responses and eye movements, participants sat comfortably approximately $75 \mathrm{~cm}$ from the computer monitor (9.25\% of visual angle for both pictures). Pupillary responses and eye movements were recorded at $300 \mathrm{~Hz}$ using an infrared eye tracker (Tobii-TX300; Tobii, Danderyd, Sweden). Prior to the start of the task, participants' gaze fixations were calibrated using a standard 9-point calibration sequence that covered all four corners and midpoints at the top, middle, and lower portion of the screen. Pupillary responses and eye movements were recorded on a trial-by-trial basis.

Heart rate variability (HRV) was measured beat to beat with a telemetric heart-rate monitor (POLAR S810). HRV was measured during the 20 minutes of baseline ( $\mathrm{T}_{\text {baseline, }}$ participants were asked to relax), during the two blocks of positive social feedback and the two blocks of negative social feedback (each block lasts about 4 minutes; HRV was calculated over the two blocks with overall positive social feedback and over the two blocks with overall negative social feedback), and during a 15 minutes postparadigm rest period $\left(\mathrm{T}_{\text {post }}\right)$.

\section{Data analytic plan}

Mood A repeated measures MANOVA with Time $\left(\mathrm{T}_{\text {pre, }}, \mathrm{T}_{\text {post }}\right)$ was performed with the different mood subscales as dependent variables.

Gaze data Eye-tracking data were analyzed over the $6,000 \mathrm{~ms}$ period that the feedback was presented onscreen. Three areasof-interest (AOIs) were defined: photograph of the self, photograph of the evaluator, and the text. By including this last AOI in the analyses, we controlled for gazes to look away from both themselves and the person that rejected them. A fixation was defined as a condition in which the eye remained stationary on a predetermined AOI (evaluator, self, or text) for a time interval of $100 \mathrm{~ms}$ or more (Wadlinger \& Isaacowitz, 2008). In line with recent research, the following dependent variables were calculated (e.g., Duque et al., 2014): (1) Fixation frequency - The number of times a participant directs (redirects) attention to a particular AOI, and is an index of participant's scanning pattern and attentional shifts. (2) Fixation time (or dwell time)-This is the total duration (in $\mathrm{ms}$ ) a participant has fixated within the boundaries of a particular AOI during a particular social feedback (positive or negative), independent of attentional shifts (e.g., number of fixations). The total fixation time for each AOI was generated by summing up fixation times for each AOI for the positive and negative feedback trials. Because some variables were not normally distributed (using the Shapiro-Wilk test), all variables were $\log$-transformed $(\log 10)$. To control for multiple comparisons in the eye-tracking indices, a $2 \times 3$ repeated measures MANOVA with Feedback (positive, negative) $\mathrm{x}$ $A O I$ (evaluator, self, text) as within factors was performed with both gaze indices as dependent variables. Significant effects were followed up by paired $t$ tests (two-tailed).

Pupillary responses Individual data were first scanned for overall data quality. All participants' data contained more than $75 \%$ of valid pupillary responses across the whole experiment. Blinks, missing, and invalid data points were first linearly interpolated using the interp1 function in MATLAB (MATLAB 7.11.0). The data were then detrended (to remove slow irrelevant drifts) with simple linear regression within each block. Pupillary responses for each of the conditions of interest (positive and negative social feedback) were calculated by subtracting the baseline pupil diameter (the first 50 time points $(\sim 165 \mathrm{~ms})$ from pupil diameter during the trial at each of the consecutive measurement points during the epoch. These differences were then averaged across trials and across participants, excluding trials for which $50 \%$ or more of the pupil dilation data were missing. In both positive and negative feedback conditions, all trials were retained in at least $90 \%$ of the participants. This resulted in two waveforms, each for 8 , $658 \mathrm{~ms}$ (2,600 time points), which represented averages over the length of the trial from the onset of the faces presentation until almost the end of the evaluation. The epochs were truncated at the end to omit the late time frames that contained a larger proportion of missing data.

Pupil analyses were conducted in MATLAB by contrasting mean waveforms (positive and negative feedback) at each time point along the waveform. Results report mean pupillary response in significant windows. Significant windows of the pupillary response were detected using permutation, pairedsample $t$ tests (e.g., Blair \& Karnisky, 1993; Maris \& Oostenveld, 2007). In line with Silk et al., 2012, family-wise Type I error was controlled for by using correction for multiple comparisons using the cluster thresholding method discussed in Maris \& Oostenveld (2007), using 10.000 permutation samples. A selection criterion (the critical $t$ value with degrees of freedom equal to 58 and $\alpha$ at .05, two-tailed) was predefined to select time points in each permutated sample, as well as in the original sample. The $t$ values of neighboring selected time points were then added to give $t$-sum (Blair \& Karnisky, 1993), which represented the $t$ value of this empirically generated cluster. While there could be more than one cluster in each permutated sample, only the cluster with the largest $t$-sum (smallest if $t$-sum was negative) was recorded (Maris \& Oostenveld, 2007). The $t$-sums of the 10.000 clusters were then used to define the upper and lower critical values so that the cluster level $\alpha$ was maintained at .05, two-tailed. The lower tail was equal to the 2.5 th percentile of the negative $t$ sums, and the upper tail the 97.5th percentile of the positive $t$ sums, respectively. Finally, the $t$-sums of the clusters in the original sample were compared against these critical values. 
Clusters with $t$-sums not bounded by the critical values were declared to show statistically significant differences between the waveforms of interest. The use of t-sum as compared to other clustering statistics enabled us to take into account both the height (magnitude of the $t$ values) and extent (number of contiguous time points) as a "cluster mass" (Pernet, Latinus, Nichols, \& Rousselet, 2014). Overall, such a nonparametric permutation test is data driven, implying that no a priori definition of time windows is required; it also accounts for the paired nature of the pupillary data.

Heart rate variability $(H R V)$ The heart rate data were first transmitted to a personal computer, filtered for measurement errors, and processed offline. Data were then filtered using a moderate filter power and a minimum protection zone of six beats per minute (Cottyn, De Clerq, Pannier, Crombez, \& Lenoir, 2006). After this step, the data were further analyzed with software specifically designed for advanced HRV analysis including artifact rejection at medium level (Kubios; Biosignal Analysis and Medical Imaging Group, Department of Physics, University of Kuopio, Kuipio, Finland). HRV can be described either by frequency or time domain indices. We used RMSSD (the root mean square successive difference of normal-to-normal intervals, in ms) as an index of HRV. RMSS D primarily reflects parasympathetic outflow and is one of the time domain indices recommended for mediated short-term changes in heart rate (Delaney \& Brodie, 2000; Pieper et al., 2007; Task Force, 1996).

To account for heart-rate adaptation and to remove the time that participants were filling in the questionnaires, only the last 15 (of 20) minutes of HRV measurement were taken for creating the baseline ( $\mathrm{T}_{\text {baseline }}$ ). The data of one participant were not included in the analyses due to missing values. Given nonnormality of HRV data distribution (Shapiro-Wilk test), these data were log-transformed. HRV between blocks of social feedback, as well as the baseline and post period will be compared by paired $t$ tests (two-tailed).

Correlation between different psychophysiological measures In order to investigate whether and how the different psychophysiological measures are correlated to each other, we performed Pearson correlations between the three different psychophysiological measures: Gaze indices (fixation frequency and fixation time), pupil dilation (in response to positive and negative feedback), and HRV (in blocks with overall positive and blocks with overall negative feedback).

Rumination Partial correlations were calculated to investigate a potential association between HRV blocks of overall positive/blocks of overall negative social feedback and brooding scores, controlled for the effect of tonic HRV (during baseline rest). Moreover, we performed partial correlations for HRV during the rest period following the social feedback paradigm (controlled for tonic HRV during the baseline rest period) to depressive brooding scores. Similar partial correlations were performed for the state rumination measure. Finally, Pearson correlations for the association between rumination and pupil dilation/gaze data were calculated.

\section{Results}

Overall, the cover story was well-believed as only 6 (of 59) participants expressed doubts at the end of the experiment. Analyses were done with and without these participants, but as the results remained similar, all participants were retained in the final analyses. ${ }^{2}$ Similarly, because of the high proportion of female participants in our sample $(n=49)$, analyses were performed with only female participants included, but data again remained similar. We thus included the whole sample in the statistical analyses. Finally, given the large age range of this sample (17-36 years old), it was tested whether age contributed to the results. All findings remained significant when age was taken into account, and thus this variable will no longer be discussed.

\section{Mood}

For the exact scores on the different VAS, we refer to Table 1. The MANOVA revealed differences in mood before versus after the social feedback paradigm indicating that participants were feeling more fatigued, $F(1,57)=16.42, p<.001$; being less vigorous, $F(1,57)=7.60, p<.01$; being more tense, $F(1$, $57)=20.07, p<.001$, and depressed, $F(1,57)=4.07, p<.05$, and less cheerful, $F(1,57)=5.63, p=.02$, after the experiment relative to before the social manipulation. By comparison, there were no differences in feelings of anger: $F(1,57)=$ $.75, p=.39$ (Table 2).

\section{Gaze data}

The multivariate test of the repeated measures MANOVA revealed a significant two-way interaction between Feedback x $A O I, F(4,54)=7.92, p<.001$, partial eta squared $=.37$ (also the main effect of AOI yielded a significant effect, $F(4,54)=$ $16.53, p<.001$. The univariate follow-up revealed an interaction for the within subjects variables Time $\mathrm{x} A O I$ for both gaze indices, $F_{\mathrm{S}}>13.75, p \mathrm{~s}<.001$, partial eta squared $>.19$ (see Table 3). Paired $t$ tests for fixation frequency revealed that negative (as compared to positive) feedback resulted in more fixations on their own photograph, $t(58)=5.37, p<.0001$. Positive feedback (as compared to negative feedback) resulted

\footnotetext{
${ }^{2}$ This corroborates with prior studies (e.g., Silk et al., 2012), and with studies showing that being excluded by a computer hurts as much as being excluded by real people (Zadro et al., 2004).
} 
Table 1 VAS measures $(\mathrm{cm})$ before $\left(\mathrm{T}_{\text {pre }}\right)$ and immediately $\left(\mathrm{T}_{\text {post }}\right)$ the social feedback paradigm

\begin{tabular}{lll}
\hline & $\mathbf{T}_{\text {pre }} \mathbf{M}(\boldsymbol{S D})$ & $\mathbf{T}_{\text {post }} \boldsymbol{M}(\boldsymbol{S D})$ \\
\hline Tired & $4.71(2.29)$ & $5.52(2.36)$ \\
Vigor & $4.04(2.14)$ & $3.59(1.83)$ \\
Anger & $1.15(1.47)$ & $1.31(1.72)$ \\
Tension & $3.76(2.48)$ & $2.95(2.17)$ \\
Depression & $1.83(2.16)$ & $2.93(2.38)$ \\
Cheerful & $4.74(1.93)$ & $4.29(1.84)$ \\
\hline
\end{tabular}

BOLD font, $p s<.05$

on more fixations on the photograph of the evaluator, $t(58)=$ $3.45, p=.006$. No differences between negative and positive feedback were observed for looking at the text, $p=.40$. Paired $t$ tests for fixation time (or dwell time) revealed that negative (as compared to positive) feedback resulted in higher fixation time on their own photograph, $t(58)=5.50, p<.0001$. Positive feedback (as compared to negative feedback) resulted in a higher fixation time on the photograph of the evaluator, $t(58)=3.93, p<.0001$. No differences between negative and positive feedback were observed for looking at the text, $p=$ .89. A Bonferroni correction for six comparisons over both gaze indices was applied for all significant effects.

\section{Pupil size}

The results of the permutation $t$ test are shown in Fig. 2. The pupil was significantly more dilated (as compared to baseline) when participants received negative evaluations (criticism) as compared to when they received positive evaluations (praise), indicating putative self-regulatory mechanisms to negative

Table 2 Overview of mean and standard deviation of the HRV during social feedback paradigm (highlighted in gray), together with the partial correlation coefficients of the habitual tendency of rumination during the social feedback. This difference was evident between 3,700 and 5,500 ms following the onset of the trial (e.g., $700 \mathrm{~ms}$ following the onset of the feedback).

Heart rate variability (HRV)

In line with prior research on tonic and phasic HRV, HRV during the baseline period was positively correlated to HRV during blocks of social feedback, as well as the postmeasurement, $r \mathrm{~s}(58)>.58, p \mathrm{~s}<.0001$ (see Table 2). During the task, HRV (controlled for the tonic HRV by means of a delta score) was higher in the blocks with overall negative feedback as compared to the blocks of overall positive feedback, $t(57)=2.76, p=.04$. During the postrest measurement, HRV (controlled for the HRV during the rest measurement at baseline by means of a delta score) was larger as compared to positive feedback blocks, $t(57)=6.39, p<.001$, and negative feedback blocks, $t(57)=4.40, p<.01$. A Bonferroni correction for five comparisons for both feedback blocks was applied.

Correlation between different psychophysiological measures

Pupil dilation scores (in the time frame that differentiated praise and criticism) were not correlated with each of both gaze indices, $r \mathrm{~s}<.24, p \mathrm{~s}>.07$, nor with phasic HRV during blocks of overall criticism and blocks with over praise, $r \mathrm{~s}<$ $.08, p s>.56$. On the other hand, phasic HRV during blocks of overall criticism and blocks with overall praise correlated positively with the fixation time and fixation frequency towards the evaluator after receiving positive feedback, $r \mathrm{~s}>.26, p \mathrm{~s}<$ .05 , but these correlations would not survive the correction for multiple comparisons.

different blocks of HRV registration. Significant correlations are marked with $\mathrm{a}$ *

\begin{tabular}{|c|c|c|c|c|}
\hline & \multicolumn{2}{|c|}{ HRV } & \multirow{2}{*}{$\begin{array}{c}\begin{array}{c}\text { Depressive } \\
\text { brooding }\end{array} \\
M=11.32 \\
S D=2.90\end{array}$} & \multirow{2}{*}{$\begin{array}{c}\begin{array}{c}\text { State } \\
\text { rumination }\end{array} \\
M=22.14 \\
S D=6.82\end{array}$} \\
\hline & & & & \\
\hline & $M$ & $S D$ & & \\
\hline T baseline & 1.52 & .22 & -.13 & -.01 \\
\hline $\begin{array}{c}\text { Blocks with } \\
\text { overall positive } \\
\text { feedback }\end{array}$ & 1.57 & .22 & $-.29 *$ & -.17 \\
\hline $\begin{array}{c}\text { Blocks with } \\
\text { overall negative } \\
\text { feedback }\end{array}$ & 1.60 & .25 & $-.25^{*}$ & -.22 \\
\hline T post & 1.68 & .22 & $-.31 *$ & $-.31 *$ \\
\hline
\end{tabular}

*Correlation coefficient is significant at the .05 level (two-tailed). $\mathrm{T}_{\text {baseline }}=15$ minutes rest before the start of the experiment; $\mathrm{T}_{\text {post }}=15$ minutes rest at the end of the study. Of note, the correlation with the subscale reflective pondering of the RRS yielded no significant results 
Table 3 Overview of mean and standard deviation of eye tracking indices during social feedback (positive and negative)

\begin{tabular}{|c|c|c|c|c|c|c|}
\hline & \multicolumn{3}{|c|}{ Positive social feedback (praise) } & \multicolumn{3}{|c|}{ Negative social feedback (criticism) } \\
\hline & $\begin{array}{l}\text { Evaluator } \\
\mathrm{M}(\mathrm{SD})\end{array}$ & $\begin{array}{l}\text { Self } \\
M(S D)\end{array}$ & $\begin{array}{l}\text { Text } \\
\text { M(SD) }\end{array}$ & $\begin{array}{l}\text { Evaluator } \\
\mathrm{M}(\mathrm{SD})\end{array}$ & $\begin{array}{l}\text { Self } \\
M(S D)\end{array}$ & $\begin{array}{l}\text { Text } \\
M(S D)\end{array}$ \\
\hline Fixation frequency & $3.21(1.25)$ & $2.48(1.21)$ & $3.34(1.74)$ & $2.92(1.14)$ & $2.83(1.25)$ & $3.33(1.70)$ \\
\hline Fixation time & $1,187.77(600.10)$ & $1,072.17(622.60)$ & $1,302.63(1,019.71)$ & $1,038.02(512.06)$ & $1,233.82(615.35)$ & $1,345.24(1,019.40)$ \\
\hline
\end{tabular}

Rumination

Using partial correlations to control for tonic HRV at baseline, the habitual tendency for depressive brooding was inversely correlated to HRV during blocks receiving overall negative social feedback, $r(58)=-.26, p=.05$; blocks receiving overall positive social feedback, $r(58)=-.30, p=.02$; and during the postrest measurements, $r(58)=-.32, p=.02$. Moreover, partial correlations (controlling for tonic HRV at baseline) were obtained for the state measure of rumination: MRSI scores were inversely correlated to the HRV during the postrest measurement, $r(55)=-.32, p=.02$, but not during the social feedback paradigm, $r \mathrm{~s}<.22, p \mathrm{~s}>.1$. Trait and state rumination were, on the other hand, not correlated with either pupil dilation or gaze data, $r \mathrm{~s}<.23, p \mathrm{~s}>.08$.

\section{Discussion}

Social feedback (e.g., criticism and praise) triggers self-reflective thoughts and feelings that need to be regulated to prevent maladaptive emotional responses to occur. In the current study, we used rigged social feedback to assess psychophysiological measures (visual gaze, pupil dilation, and HRV) as a proxy of regulatory responses to emotional information. Reports of mood after (as compared to before) the social feedback paradigm confirmed increased emotional reactivity (feeling more fatigue, less vigorous, more depressed, and less cheerful, although also less tensed) in response to the social feedback. Because participants were not explicitly told about the main purpose of the study, we assessed the spontaneous/intrinsic tendency of participants to regulate emotional responses triggered by the social feedback.

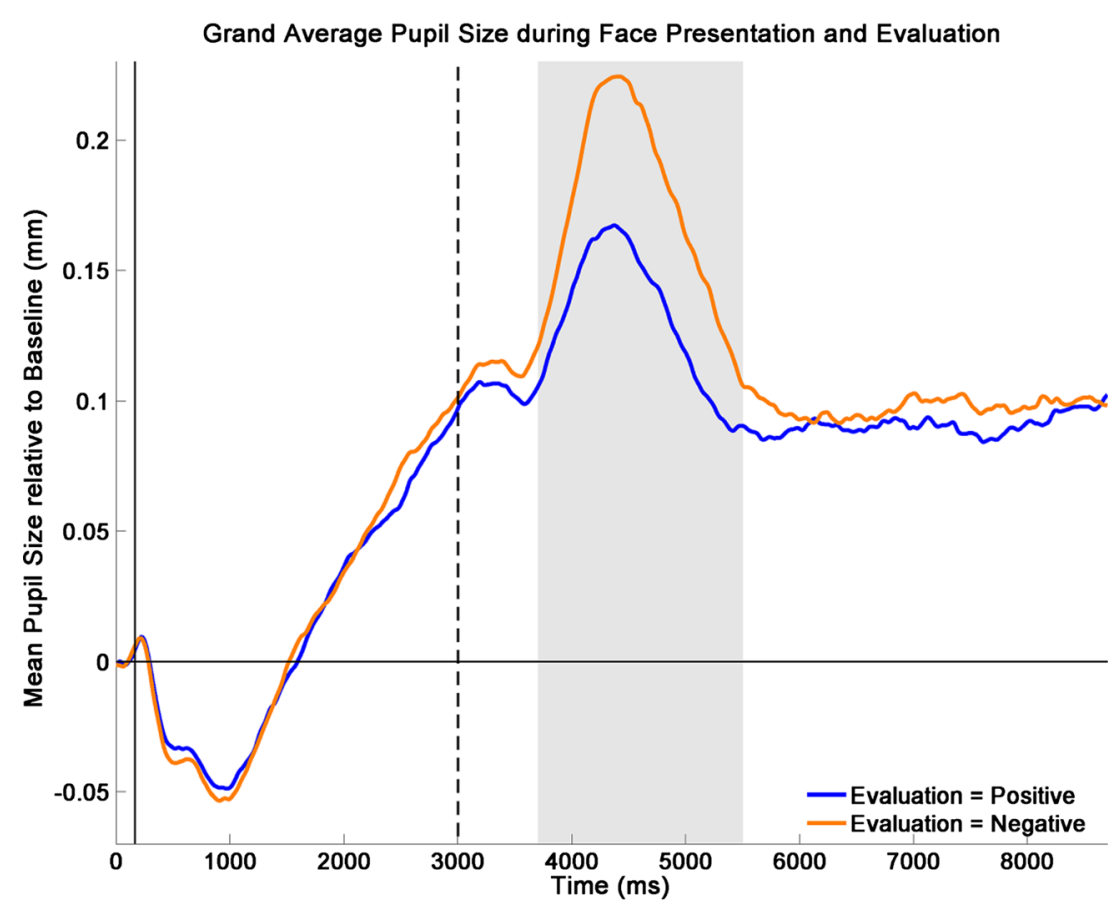

Fig. 2 Time series of pupillary response. The figure shows baseline corrected pupillary responses when the portrait photographs of the participant and evaluator are presented and (period after dotted vertical line) after social feedback was presented. The time window with significant differences between pupillary responses when receiving positive or negative evaluations, based on the Permutation $t$ test $(3,700-5,500 \mathrm{~ms})$, is highlighted in gray 
A novel aspect of this study is the examination of gaze patterns and attentional deployment in social feedback. Consistent with our expectations (e.g., Fredrickson, 1998; Mor \& Winquist, 2002), attentional preference was consistently observed toward evaluators that expressed positive social feedback (i.e., praise), but consistently toward one's own personal photograph after receiving negative social feedback (i.e., criticism). This pattern was present across both indices of gaze patterns: fixation frequency and fixation time (or dwell time). No effects of social feedback were observed for fixation frequency or fixation time towards the text, which shows that participants were not generally avoiding attention towards the photograph of the evaluator or their own photograph. All together, people show interest in the person who likes them, and this is in line with the knowledge that positive social feedback drives and forms social bonding, attachments, interactions and relationships (Baumeister \& Leary, 1995). Negative feedback, on the other hand, results in a heightened attention allocation towards their own photograph, possibly associated with a tendency of self-focused attention to reflect upon traits, thoughts, and feelings (Mor \& Winquist, 2002). Of note, prior eyetracking studies observed that (threat for) social exclusion induced attentional preference towards individuals in the environment; for example, to smiling faces that signal a higher likelihood of potential new affiliation (e.g., DeWall et al., 2009) or to players that could potentially reintegrate them in a "looking game" (e.g., Böckler, Hömke, \& Sebanz, 2014). These results have been interpreted as a coping mechanism aimed at restoring social status after rejection (Vandellen et al., 2012). However, our study is the first to compare attentional deployment toward two smiling faces, one of the participant him- or herself and the other of the person providing feedback. These attentional deployment processes inform about the focus of the emotion regulatory effort in terms of the relational functions (rejection vs. acceptance). Moreover, these attentional deployment processes are based on top-down mechanisms and not on contextual features (e.g., facial expression), and thus provide a unique contribution to the existing research on the theoretical framework of emotion regulation.

Pupil dilation (as a measure of cognitive-affective nervous system processing) and HRV (as a measure of flexible adaptation to environmental stressors) were assessed to index emotion regulatory effort following socially stressful stimuli. As expected (e.g. Silk et al., 2012), pupillary responses were larger to interpersonal negative as compared to positive feedback and this effect lasted for about two seconds. As prior research has shown that the pupillary response provides a summary measure of neural activation that reflects (1) emotional reactivity in limbic regions (Siegle et al., 2003) or (2) activation in prefrontal regions implicated in emotion regulation (Siegle et al., 2011). Moreover, Johnstone et al. (2007) demonstrated that in healthy controls emotion regulation effort was associated with greater pupil dilation and decreased activation in limbic areas. Greater pupil dilation (especially following negative social feedback) might thus reveal an adaptive regulation towards emotional distress.

Regarding the cardiac response, larger HRV (both tonic and phasic) has been linked to flexible and adaptive emotion regulation, while lower HRV has been taken to reflect a rigid and hyper-vigilant response to emotional distress (Brosschot, Van Dijk, \& Thayer, 2007; Thayer et al., 2012; Thayer \& Lane, 2000; Thayer et al., 2009). In the present study, HRV was larger in blocks with overall negative feedback as compared to blocks with overall positive feedback. Moreover, HRV was larger "during" and "after" as compared to "before" receiving social feedback. These HRV results suggest that social feedback provoked a regulatory response to selfrelevant information that triggers an affective response. Interestingly, the more participants reported to use depressive brooding in daily life, the lower HRV during blocks receiving overall positive and overall negative social feedback. In other words, depressive brooding - considered an inflexible and preservative cognitive style - is associated with the flexibility in emotional responses required for both positive and negative social feedback, possibly to prevent those responses to become maladaptive or socially inadequate. In addition, this inverse association between HRV and rumination also extended to the rest period at the end of the study, both for the habitual tendency of depressive brooding as well as for state rumination during the rest period. Prior research of Beevers, Ellis, and Reid (2011) demonstrated that low HRV during a sad mood provocation was associated with the activation of dysfunctional attitudes following a stressful situation, suggesting lower HRV a physiological marker of cognitive reactivity and depression vulnerability. Altogether, HRV results suggest that healthy participants show enhanced regulatory control when confronted with both positive and negative emotionally potent stimuli, most likely because they are flexibly and adaptively regulating their emotions triggered by environmental stressors.

Despite the interesting and novel findings, some limitations deserve discussion. First, because we did not want to compromise on ecological validity of receiving first impressions, selfreport measures of how participants felt during the social feedback paradigm were not obtained (only before vs. after the paradigm). This way, we have no subjective information on the emotional reactivity specifically to positive and negative feedback. Nevertheless, our physiological measures of HRV and pupil dilation suggest increased emotional reactivity during the social feedback (most pronounced after receiving negative feedback) that possibly induced some self-regulatory processes to cope with the feedback. Second, participants may have reacted differently to negative social feedback based on the attractiveness of the evaluator, as the attractiveness of a face has been found to influence neural activity in reward related regions (O’Doherty et al., 2003). However, we did 
not obtain such data, and further studies should take this variable into account. Last, the unbalanced distribution of males and females challenges the generalizability of the results to both genders. Given the small number of males, the results might especially refer to female participants given that the results remained stable when analyses were rerun without the males. Moreover, prior meta-analyses have documented a strong relation between self-focus and negative affect in female-dominated samples (Mor \& Winquist, 2002) and larger effect sizes for changes in mood as a function of social exclusion for female participants (Blackhart et al., 2009). Future research should aim to investigate the specificity of such effects across genders.

To conclude, the current findings reveal that - in healthy volunteers - the focus of attention is associated with the type of feedback: a preferential focus on the evaluator when the feedback was positive (i.e., praise) but a preferential self-focus when the feedback was negative (i.e., criticism). In addition, they display larger pupil dilation and HRV in response to negative as compared to positive social feedback, possibly reflecting enhanced regulatory —control to cope with emotionally potent events.

Acknowledgments MAV (FWO08/PDO/168) is a postdoctoral fellow of the Research Foundation Flanders (FWO). Preparation of this paper was also supported by Grant BOF10/GOA/014 for a Concerted Research Action of Ghent University (awarded to RDR). The authors declare no conflicts of interest.

\section{References}

Aldao, A., Mennin, D. S., \& McLaughlin, K. A. (2013). Differentiating worry and rumination: Evidence from heart rate variability during spontaneous regulation. Cognitive Therapy and Research, 37(3), 613-619. doi:10.1007/s10608-012-9485-0

Appelhans, B. M., \& Luecken, L. J. (2006). Heart rate variability as an index of regulated emotional responding. Review of General Psychology, 10, 229-240. doi:10.1037/1089-2680.10.3.229

Baumeister, R. F., \& Leary, M. R. (1995). The need to belong: Desire for interpersonal attachments as a fundamental human motivation. Psychological Bulletin, 117, 497-529.

Beevers, C. G., Ellis, A. J., \& Reid, R. M. (2011). Heart rate variability predicts cognitive reactivity to a sad mood provocation. Cognitive Therapy \& Research, 35, 395-403.

Blackhart, I. C., Nelson, B. C., Knowles, M. L., \& Baumeister, R. F. (2009). Rejection elicits emotional reactions but neither causes immediate distress nor lowers self-esteem: A meta-analytic review of 192 studies on social exclusion. Personality and Social Psychology Review, 13, 269-309.

Blair, R. C., \& Karniski, W. (1993). An alternative method for significance testing of waveform difference potentials. Psychophysiology, $30,518-524$.

Böckler, A., Hömke, P., \& Sebanz, N. (2014). Invisible man: Exclusion from shared attention affects gaze behavior and self-reports. Social Psychological and Personality Science, 5(2), 140-148.

Bradley, M. M., Miccoli, L., Escrig, M. A., \& Lang, P. J. (2008). The pupil as a measure of emotional arousal and autonomic activation.
Psychophysiology, 45, 602-607. Retrieved from http://dx.doi.org/ 10.1111/j.1469-8986.2008.00654.x

Brosschot, J. F., van Dijk, E., \& Thayer, J. F. (2007). Daily worry is related to low heart rate variability during waking and the subsequent nocturnal sleep period. International Journal of Psychophysiology, 63, 39-47.

Cottyn, J., De Clercq, D., Pannier, J. L., Crombez, G., \& Lenoir, M. (2006). The measurement of competitive anxiety during balance beam performance in gymnasts. Journal of Sports Sciences, 24(2), $157-164$.

Critchley, H. D., Tang, J., Glaser, D., Butterworth, B., \& Dolan, R. J. (2005). Anterior cingulate activity during error and autonomic response. NeuroImage, 27, 885-895.

Delaney, J. P. A., \& Brodie, D. A. (2009). Effects of short-term psychological stress on the time and frequency domains of heart-rate variability. Perceptual and Motor Skills, 91(2), 515-524.

DeWall, C. N., Maner, J. K., \& Rouby, D. A. (2009). Social exclusion and early-stage interpersonal perception: Selective attention to signs of acceptance. Journal of Personality and Social Psychology, 96, 729741. doi: $10.1037 / \mathrm{a} 0014634$

Duque, A., Sanchez, A., \& Vazquez, C. (2014). Gaze-fixation and pupil dilation in the processing of emotional faces: The role of rumination. Cognition \& Emotion. doi:10.1080/02699931.2014.881327

Fredrickson, B. L. (1998). What good are positive emotions? Review of General Psychology, 2, 300-319.

Gross, J. J., \& Thompson, R. A. (2007). Emotion regulation: Conceptual foundations. In J. J. Gross (Ed.), Handbook of emotion regulation (pp. 3-24). New York, NY: Guilford Press.

Hermans, D., \& Dehouwer, J. (1994). Affective and subjective familiarity ratings of 740 Dutch words. Psychologica Belgica, 34(2/3), 115139.

Johnstone, T., van Reekum, C. M., Urry, H. L., Kalin, N. H., \& Davidson, R. J. (2007). Failure to regulate: Counterproductive recruitment of top-down prefrontal-subcortical circuitry in major depression. Journal of Neuroscience, 27, 8877-8884. doi:10.1523/ JNEUROSCI.2063-07.2007

Koval, P., Ogrinz, B., Kuppens, P., Van den Bergh, O., Tuerlinckx, F., \& Sütterlin, S. (2013). Affective instability in daily life is predicted by resting heart rate variability. PLoS One, 8(11), e81536. doi:10.1371/ journal.pone. 0081536

Leary, M. R., Koch, E. J., \& Hechenbleikner, N. R. (2001). Emotional responses to interpersonal rejection. In M. Leary (Ed.), Interpersonal rejection (pp. 145-166). New York, NY: Oxford University Press.

Maris, E., \& Oostenveld, R. (2007). Nonparametric statistical testing of EEG- and MEG-data. Journal of Neuroscience Methods, 164, 177190.

McCormack, H. M., David, J., Horne, L., \& Sheater, S. (1988). Clinical applications of visual analogue scales: A critical review. Psychological Medicine, 18, 1007-1019. Retrieved from http:// dx.doi.org/10.1017/S0033291700009934

Mor, N., Marchetti, I., \& Koster, E. H. W. (2015). A new state measure of self-reflection: Psychometric evaluation of the Momentary Ruminative Self-focus Inventory (MRSI). Manuscript submitted for publication.

Mor, N., \& Winquist, J. (2002). Self-focused attention and negative affect: A meta-analysis. Psychological Bulletin, 128, 638-662.

O’Doherty, J., Winston, J., Critchley, H., Perrett, D., Burt, D. M., \& Dolan, R. J. (2003). Beauty in a smile: The role of medial orbitofrontal cortex in facial attractiveness. Neuropsychologia, 41, 147-155.

Park, G., Vasey, M. W., Van Bavel, J. J., \& Thayer, J. F. (2014). When tonic cardiac vagal tone predicts changes in phasic vagal tone: The role of fear and perceptual load. Psychophysiology, 51(5), 419-426. doi:10.1111/psyp.12186 
Pernet, C. R., Latinus, M., Nichols, T. E., \& Rousselet, G. A. (2014). Cluster-based computational methods for mass univariate analyses of event-related brain potentials/fields: A simulation study. Journal of Neuroscience Methods. doi:10.1016/j.jneumeth.2014.08.003

Pieper, S., Brosschot, J. F., van der Leeden, R., \& Thayer, J. F. (2007). Cardiac effects of momentary assessed worry episodes and stressful events. Psychosomatic Medicine, 69, 901-909.

Rottenberg, J. (2007). Cardiac vagal control in depression: A critical analysis. Biological Psychology, 74, 200-211.

Siegle, G. J., Steinhauer, S. R., Friedman, E. S., Thompson, W. S., \& Thase, M. E. (2011). Remission prognosis for cognitive therapy for recurrent depression using the pupil: Utility and neural correlates. Biological Psychiatry, 69, 726-733.

Siegle, G. J., Steinhauer, S. R., Stenger, V. A., Konecky, R., \& Carter, C. S. (2003). Use of concurrent pupil dilation assessment to inform interpretation and analysis of fMRI data. NeuroImage, 20, 114-124.

Siegle, G. J., Steinhauer, S. R., Thase, M. E., Stenger, A., \& Carter, C. S. (2002). Can't shake that feeling: Event-related fMRI assessment of sustained amygdala activity in response to emotional information in depressed individuals. Biological Psychiatry, 51, 693-707.

Silk, J. S., Stroud, L. R., Siegle, G. J., Dahl, R. E., Lee, K. H., \& Nelson, E. E. (2012). Peer acceptance and rejection through the eyes of youth: Pupillary, eye-tracking and ecological data from the Chatroom Interact task. Social Cognitive \& Affective Neuroscience, 7, 93-105.

Task Force of the European Society of Cardiology the North American Society of Pacing and Electrophysiology. (1996). Heart rate variability: Standards of measurement, physiological interpretation, and clinical use. Circulation, 93(5), 1043-1065. doi:10.1161/01.cir.93. 5.1043

Thayer, J. F., Åhs, F., Fredrikson, M., Sollers, J. J., \& Wager, F. D. (2012). A meta-analysis of heart rate variability and neuroimaging studies: Implications for heart rate variability as a marker of stress and health. Neuroscience and Biobehavioral Reviews, 36, 747-756.

Thayer, J. F., Hansen, A. L., Saus-Rose, E., \& Johnsen, B. H. (2009). Heart rate variability, prefrontal neural function, and cognitive performance: The neurovisceral integration perspective on self-regulation, adaptation, and health. Annals of Behavioral Medicine, 37, 141-153.

Thayer, J. F., \& Lane, R. D. (2000). A model of neurovisceral integration in emotion regulation and dysregulation. Journal of Affective Disorder, 61, 201-216. doi:10.1016/S0165-0327(00)00338-4
Treynor, W., Gonzalez, R., \& Nolen-Hoeksema, S. (2003). Rumination reconsidered: A psychometric analysis. Cognitive Therapy and Research, 27, 247-259. doi:10.1023/A:1023910315561

Urry, H. L., van Reekum, C. M., Johnstone, T., Kalin, N. H., Thurow, M. E., Schaefer, H. S., \& Davidson, R. J. (2006). Amygdala and ventromedial prefrontal cortex are inversely coupled during regulation of negative affect and predict the diurnal pattern of cortisol secretion among older adults. Journal of Neuroscience, 26, 4415-4425.

Urry, H. L., van Reekum, C. M., Johnstone, T., \& Davidson, R. J. (2009). Individual differences in some (but not all) medial prefrontal regions reflect cognitive demand while regulating unpleasant emotion. NeuroImage, 47, 852-863.

van Reekum, C. M., Johnstone, T., Urry, H. L., Thurow, M. T., Schaefer, H. S., Alexander, A. L., \& Davidson, R. J. (2007). Gaze fixations predict brain activation during the voluntary regulation of pictureinduced negative affect. NeuroImage, 36, 1041-1055. Retrieved from http://dx.doi.org/10.1016/j.neuroimage.2007.03.052

Van Steenbergen, H., \& Band, G. P. H. (2013). Pupil dilation in the Simon task as a marker of conflict processing. Frontiers in Human Neuroscience, 7(215). doi.10.3389/fnhum.2013.00215

Vandellen, M., Knowles, M. L., Krusemark, E., Sabet, R. F., Campbell, W. K., McDowell, J. E., \& Clementz, B. A. (2012). Trait self-esteem moderates decreases in self-control following rejection: An information-processing account. European Journal of Personality, 26, 123-132. doi:10.1002/per.1845

Wadlinger, H. A., \& Isaacowitz, D. M. (2008). Looking happy: The experimental manipulation of a positive visual attention bias. Emotion, 8, 121-126.

Wirth, J. H., Sacco, D. F., Hugenberg, K., \& Williams, K. D. (2010). Eye gaze as relational evaluation: Averted eye gaze leads to feelings of ostracism and relational devaluation. Personality and Social Psychology Bulletin, 36, 869-888.

Woody, M. L., McGeary, J. E., \& Gibb, B. E. (2014). Brooding rumination and heart rate variability in women at high and low risk for depression: Group differences and moderation by COMT Genotype. Journal of Abnormal Psychology, 123(1), 61-67.

Zadro, L., Williams, K. D., \& Richardson, R. (2004). How low can you go? Ostracism by a computer is sufficient to lower self-reported levels of belonging, control, selfesteem, and meaningful existence. Journal of Experimental Social Psychology, 40, 560-567. 